\title{
Beyond screening: a review of pediatric primary care models to address maternal depression
}

\author{
Nomi S. Weiss-Laxer ${ }^{1}$, Rheanna Platt ${ }^{2}$, Lauren M. Osborne ${ }^{3}$, Mary Kimmel ${ }^{4}$, Barry S. Solomon ${ }^{5}$, Tamar Mendelson ${ }^{6}$, \\ Lindsey Webb ${ }^{6}$ and Anne W. Riley ${ }^{1}$
}

Depression is one of the most debilitating chronic disorders in the United States, affecting 15 million children in homes with depressed mothers, many of whom endure household chaos, inconsistent nurturing, inadequate safety practices, and harsh discipline. Depressed mothers are under diagnosed and undertreated, yet there is broad consensus about the importance of identifying and managing maternal depression, as reflected in recommendations by pediatric and obstetric professional organizations to routinely screen for perinatal depression. Screening was shown to be acceptable to women and most pediatric providers, and adding a screening component need not impair clinic efficiency. Screening, however, is not sufficient, and there are few models in the literature to guide medical practices in implementing successful interventions to identify, treat, and prevent maternal depression, particularly in the pediatric setting. We reviewed the literature and identified six studies that evaluated models for screening and managing mothers' depression in pediatric primary care settings. Some of these interventions have promise, but no studies characterized health outcomes of the depressed mothers and children. We discuss the components of these models, their implementation, and the practice and research needed to create effective pediatrics-based systems to reduce the negative effects of maternal depression on mothers, children, and families.

D epression is one of the most debilitating chronic disorders in the United States (1), a fact experienced every day by the 15 million children being raised in homes with depressed mothers (2). Children living with depressed mothers may experience household chaos, inconsistent nurturing, inadequate safety practices, harsh discipline, and child maltreatment $(3,4)$. Compared to nonexposed peers, children of depressed mothers are two to three times more likely to develop psychiatric disorders themselves and to do poorly in school (5-9). Moreover, recent studies suggest that remission of maternal depression is associated with significant improvement in child behavioral and mental health outcomes (10). Children's home, family, and social environments appear to explain the majority of the risk for the intergenerational transmission of depression (11), with genetics contributing less than half of the risk $(10,12)$. It is the immediate family environment, what Bronfenbrenner refers to as "proximal processes," that powerfully shapes children's neural, cognitive, social, and behavioral development (13). Thus, it is imperative to promote the safety and wellbeing of children living in homes with depressed mothers.

According to the 2009 US National Academies of Science review of maternal depression, "Few opportunities exist to identify the vulnerable population of children living in households with one or more parents experiencing depression or to offer prevention and treatment services that can improve the care of the depressed parent in a framework that also offers services for children" (2). The pediatric primary care setting provides a unique opportunity for the provision of such services, particularly in the current era, which promotes patient and family-centered care. Recognizing this potential, the American Academy of Pediatrics recommends incorporating screening and referral for maternal depression into routine pediatric practice (14). There is broad consensus that screening for maternal depression in pediatrics is acceptable to most women (15) and many pediatric providers (16), and there is good evidence that routine screening does not necessarily impair clinic efficiency (17-21). Screening, however, is not sufficient to promote maternal and child well being (22). Several primary-care-based studies have shown that screening alone does not improve outcomes and that screening programs need to be linked to effective management of maternal depression (including referral when necessary, effective hand-offs to mental health providers, and tracking and follow-up of maternal and child outcomes).

As evidenced by two recent reviews, research supports the efficacy of prevention and treatment of perinatal depression, both with antidepressant medication and psychotherapy $(23,24)$. It should be noted however, that most of these clinical

\footnotetext{
'Department of Population, Family and Reproductive Health, Johns Hopkins Bloomberg School of Public Health, Baltimore, Maryland; ${ }^{2}$ Department of Psychiatry and Behavioral Sciences, Division of Child and Adolescent Psychiatry, Johns Hopkins University School of Medicine, Baltimore, Maryland; ${ }^{3}$ Department of Psychiatry and Behavioral Sciences, Women's Mood Disorders Center, Johns Hopkins University School of Medicine, Baltimore, Maryland; ${ }^{4}$ Department of Psychiatry, University of North Carolina, Chapel Hill, North Carolina; ${ }^{5}$ Department of Pediatrics, Division of General Pediatrics and Adolescent Medicine, Johns Hopkins School of Medicine, Baltimore, Maryland; ${ }^{6}$ Department of Mental Health, Johns Hopkins Bloomberg School of Public Health, Baltimore, Maryland. Correspondence: Nomi S. Weiss-Laxer (Nweissl1@jhu.edu)

Received 18 April 2015; accepted 30 September 2015; advance online publication 18 November 2015. doi:10.1038/pr.2015.214
} 


\section{Review | weiss-Laxer etal.}

trials were not conducted in primary care, and that there is a significant attrition from sites of detection and referral to attendance and engagement in specialty mental health settings (25). Moreover, the clinical trials in the two reviews only evaluated efficacy, but the effectiveness of such programs delivered in the "real world" has yet to be demonstrated.

In a recent review of postpartum depression screening and management programs across settings, both United States and international (from pediatrics, primary care-, and communitybased), only a fraction were shown to improve maternal outcomes (26). This review highlighted multiple systems-level barriers to getting women into treatment including requirements for psychiatric evaluations, often off-site, before treatment is provided (26). To date, much of this work to "bridge the gap" between efficacy and effectiveness has been carried out in obstetrics or community medical settings caring for adults. However, recent studies indicate that many women do not attend obstetric or medical visits after birth. Even for mothers with medically complex pregnancies, only half to two-thirds attend obstetric visits within 3 mo of delivery or make a primary care visit within $1 \mathrm{y}$ of delivery (27). Therefore, bridging the efficacy to effectiveness gap for mothers of young children is likely to require interventions in pediatric settings. Identifying and addressing maternal depression in pediatrics, however, involves training, ethical, legal, and insurance challenges because mothers are not the identified patients of pediatric providers $(28,29)$.

In order to better understand the current practices and optimal strategies for managing maternal depression, it is critical to characterize the models that have been evaluated. We conducted a comprehensive review of the literature evaluating maternal depression intervention models located primarily or solely within pediatric primary care settings that integrate referral and/or management with screening for maternal depression. In this article we seek to: (i) describe recently published models and their core components, (ii) discuss barriers and facilitators to implementing such models, and (iii) highlight future practices and research needed to reduce the effects of maternal depression on mothers, families, and children.

\section{METHODS}

We conducted a systematic search of the scientific literature on maternal depression management models in pediatrics, searching three databases, PubMed, Cochrane, and Embase in March 2015. We focused on: (i) maternal depression, (ii) pediatrics, and (iii) screening and management, using a combination of controlled vocabulary (e.g., MeSH) and key word terms. We included only English-language articles published in peer-reviewed journals. Articles were required to report an evaluation of a maternal depression management model delivered through a primary care pediatrics practice. Interventions that only included a screening program were excluded. Studies were included only if they involved at least one element for depression management such as referral for evaluation or treatment, consultation, coordination of care, or follow-up. PubMed and EMBASE records were limited to the past $15 \mathrm{y}$ to ensure comparability of practices and with current practice. All studies reviewed for this paper were publically available. After duplicate records were removed, two authors scanned titles (R.P. and N.S.W.-L.) and four reviewed abstracts (L.M.O., B.S.S., R.P., and N.S.-W.L.) for relevancy. All authors evaluated the full text versions of remaining articles for inclusion. Disagreements were resolved through a consensus process. Additional papers were identified from cross-checking reference lists from relevant empirical articles (L.W.). R.P. and N.S.W.L. abstracted the final sample into Table 1.

\section{RESULTS}

Our initial scientific literature search yielded 2,850 records (see Figure 1). After duplicates were deleted, 2,310 remained. From those, all except 93 articles were excluded based on title scanning and abstract review. After a full text review of these articles, only five studies described models and preliminary outcomes of maternal depression management interventions. One additional study, published in May 2015 was identified after the search (30). Five of the six models reviewed were from the United States, and one was from Greece.

Table 1 summarizes the six models, their components, and their reported outcomes. All studies had the goal of improving detection and management of mothers' depressive symptoms. Yet, reflective of the state of this science in pediatrics, maternal depression outcomes and child health outcomes were not reported. Hence, we focus on the design and components of the programs and describe the outcomes they do present, including screening and referral rates, provider knowledge, patient engagement, and patient satisfaction.

Agapidaki et al. (31) describe an intervention based on the Health Belief Model and Social Ecological Theory. The intervention involved physician self-directed learning from a toolkit that included information about maternal depression, guidelines for screening, the Patient Health Questionnaire-2 (PHQ2), a list of community resources for referral, and a screening reminder to be placed in the office (31). Chaudron et al. (28) reported on a universal maternal depression screening program in a university-based pediatric primary care clinic. The intervention consisted of group informational meetings for pediatric residents and nurses regarding implementation of standardized screening, with guidelines for referral to the clinic on-site social worker (28). Wichman et al. (32) evaluated a universal screening program within a collaborative care program in a "Family Clinic" employing physicians board certified in family medicine, internal medicine, or pediatrics. As reviewed elsewhere (33), collaborative care typically involves development of a care plan within primary care in consultation with a psychiatrist, and comprehensive follow up with a care manager. The Wichman program included nurse-administered screening for depression among mothers of young infants. The nurse scored and communicated the depression score to the physician, as well as the answer to the question on suicidality. The provider determined whether intervention was necessary, including whether to contact the depression care manager. The care manager reviewed all screening 


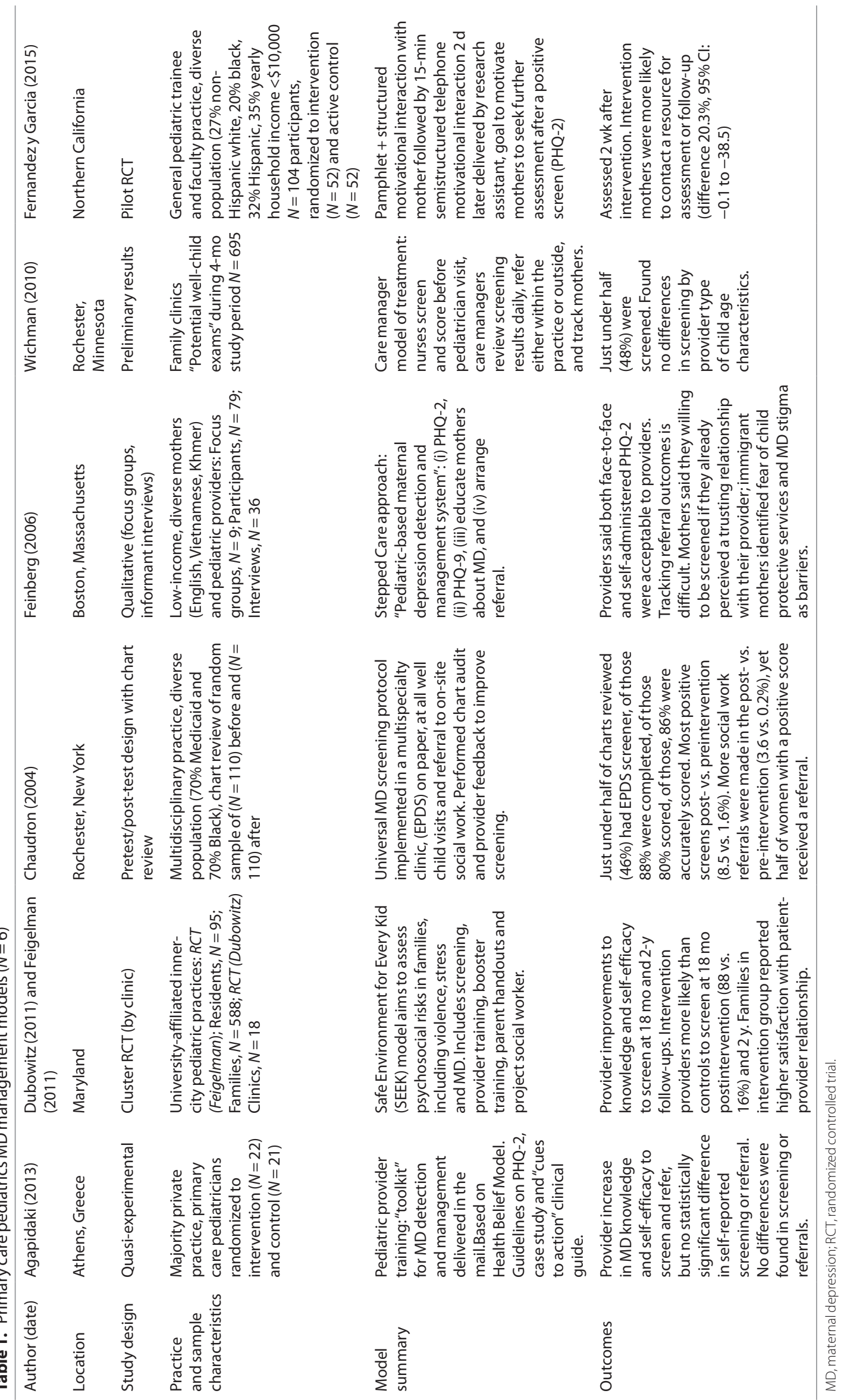




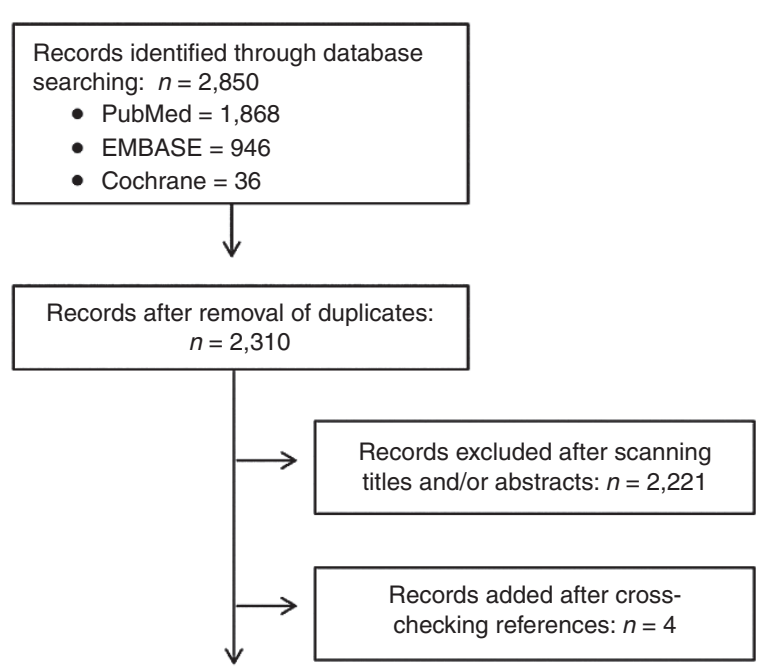

Full-text articles assessed: $n=93$
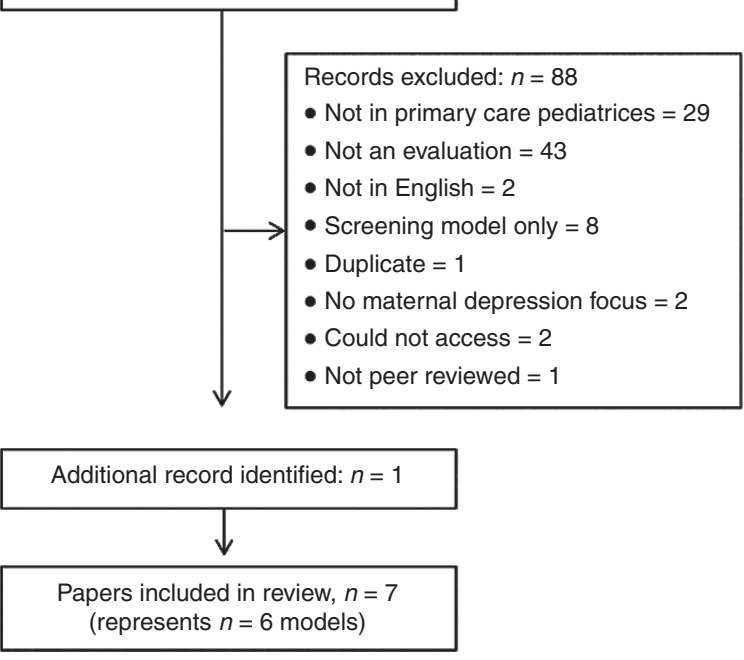

Figure 1. Flow chart of literature screening and selection process.

forms daily and notified mother's primary care doctor of positive results. The care manager coordinated mothers' care with internal and community resources. This model included an on-call psychiatry consultant and family medicine consultants. Feinberg et al. $(34,35)$ describe provider and patient acceptability of a Pediatric-Based Maternal Depression Detection and Management System in five community health centers in Boston. Their model is based on stepped care principles, i.e., that different individuals require different levels of care and that each person should be matched to the appropriate level of care. All clinics conducted universal depression screening of mothers attending well child visits, assessment of additional symptoms, level of impairment and suicide risk, provided educational discussions on depressive symptoms, parenting and self-management, and guidance regarding follow-up care. Staff training was provided, addressing maternal depression epidemiology, typical case presentation, use of management tools, and procedures for crisis situations (34). Each clinic had systems to link mothers with primary care, behavioral health and other services. Dubowitz et al. and Feigelman et al. described the Safe Environment for Every Kid (SEEK) model $(36,37)$. SEEK includes training child health primary care professionals to briefly assess and to help address targeted psychosocial problems, and training on some, like maternal depression, that pediatricians may be less knowledgeable about. It also includes several hour-long provider trainings on social determinants of child health, patient education handouts and provider training booster sessions. Referrals are made to a dedicated project social worker who helps families manage identified psychosocial risks. Fernandez y Garcia et al. (30) describe the Motivating Our Mothers (MOM) trial. MOM included officebased written and verbal depression education to mothers who screened positive on the PHQ-2, followed by a 15-min phone booster session, focused on enhancing maternal motivation to seek additional help (assessment or treatment) at one of six local resources.

These few well-described models for managing mothers' depression in pediatric settings were all designed for active pediatric practices, and several also had access to adult specialists (e.g., internist or family practitioners) on-site. The programs vary in intensity, theoretical orientation, and the breadth of provider education. Only two of the six interventions $(30,31)$ did not include an on-site mental health professional (e.g., social worker or care manager) to whom the provider could refer women who screened positive (Fernandez y Garcia et al. did have a trained research assistant who provided follow up). While the intervention described in Agapidaki et al. increased provider knowledge and self-efficacy, it did not increase screening and referral, which the authors suggested may have been related to lack of confidence in or coordination with local mental health providers. Although the other four programs had on-site social workers or care coordinators, their presence did not guarantee screening would occur: two models that implemented universal maternal depression screening protocols still found that less than half of mothers were screened for depression $(28,32)$. The failure to screen more than half the mothers of pediatric patients in these relatively well-resourced "universal" screening programs highlights the challenges to implementing protocols for addressing maternal mental health, unrelated to the efficacy of screening, or depression treatment.

The program approaches and reported outcomes ranged from improving provider knowledge of psychosocial risk, to screening rates to patient satisfaction. Pediatric residents in the SEEK model received an 8-h group training session as well as a 1-h booster session, in addition to receiving written materials, all in the context of a universal screening for family risks. In contrast, the Agapidaki model only included mailed training materials to providers. The SEEK model sustained provider knowledge effects up to 2 y $(37,38)$. The Agapidaki model did not show differences between the control and the intervention groups in terms of screening or referral practices, although these rates were self-reported (31). SEEK, on the other hand, reported a large difference in documented screening frequency $18 \mathrm{mo}$ after the provider education 
intervention; $88 \%$ of patients' mothers compared to $16 \%$ in the control group (37). And, lastly, the stepped care approach outlined by Feinberg et al. is promising based on good acceptability and feasibility in interviews $(34,35)$. These also characterized mothers' concerns regarding fear of stigma and child protective service involvement (among immigrant mothers in particular). Their results regarding effectiveness are not yet published $(34,35)$.

\section{DISCUSSION}

This paper identified and reviewed current literature describing maternal depression intervention models in pediatric primary care that go beyond screening for maternal depression and implement a management protocol. Program models varied in terms of whether they included provider training, supports for referral, consultation, and/or follow-up. Only six models met our search criteria by providing sufficient description of the intervention and at least preliminary outcomes. None reported maternal or child health outcomes such as maternal depression symptoms or child's behavior. Study outcomes included the proportion of mothers screened, the proportion referred to care, provider knowledge change, patient satisfaction, and patient engagement.

\section{Barriers to Depression Detection and Management in Pediatric Settings}

The lack of published literature is not surprising given the array of potential physician, patient, practice, and systems-level barriers to addressing mothers' needs in the pediatric setting. With respect to provider and practice-level barriers, in 2002, Olson et al. found that nearly $60 \%$ of surveyed pediatricians felt responsible for recognizing maternal depression, yet they identified a range of barriers to maternal depression management, including lack of time in the visit and insufficient training or preparation to diagnose, counsel or treat mothers with depression (16).

While pediatrics residency training has included greater emphasis on the psychosocial aspects of children's lives over the past decade, there continues to be a paucity of training on maternal depression and adult mental health issues. Based on a national survey of pediatricians in the American Academy of Pediatrics, Head et al. (39) reported that pediatricians with more training and experience in adult mental health issues, those who used systematic identification methods (i.e., screening tools), and those who believed mental health services were available in their communities were less likely to report barriers to depression management. Unfortunately, $80 \%$ of pediatric resident respondents reported not receiving any training in techniques to manage adult mental health problems.

With respect to practice-level barriers, developing and maintaining effective referral tracking systems (as has been done in adult collaborative care programs) appears to be important but underutilized. Only one of the models in our review, the Care Manager Model, had a staff member dedicated to patient screening, referrals and entry to mental health care (32). The importance of follow-up and tracking was also highlighted in the Fernandez y Garcia model, which demonstrated a promising effect of targeted outreach and follow up with screen positive mothers with respect to initiation of help seeking.

Based on our review, models that addressed the following barriers were most successful at engaging mothers in screening and completing referrals: engaging physicians and/or mothers in meaningful education; expecting universal screening with easy access to screeners; and easy linkage to colocated or community-based care managers or social workers to facilitate a smooth referral process. With the increasing use of electronic medical records, physician prompts, and screening tools can be successfully integrated into routine care, as the electronic medical records can facilitate maternal depression identification and linkage to care, and tracking of referrals. In a randomized trial, Carroll et al. (40) demonstrated higher identification and referral rates for pediatricians whose patients automatically received a prescreening and "just in time" delivery of referral materials than for pediatricians who were given a reminder to screen during the appointment. Billing for maternal mental health treatment in pediatric primary care and funding for colocated care coordinators, case managers, and mental health providers remain major systems-level barriers to effective implementation.

\section{Lessons in Maternal Depression Management to Be Learned} From Nonpediatric Settings

Although research in this area is just beginning in pediatrics, we can learn from maternal depression management systems in obstetrics and family medicine settings where numerous intervention studies have shown efficacy (41-49). These clinics vary in the types of providers they employ, from obstetricians to mother-only general practitioners to maternal-child clinics but all differ from the pediatric models in that the providers are already the women's providers (as opposed to her children's). Nevertheless, these models contain elements that may be useful in pediatric practices.

Several studies describe models for managing depression in maternal health care settings (42-45,50,51). Miller et al., working with a largely Hispanic clientele in an urban community health center, trained providers in screening, assessment, and treatment engagement strategies, with care provided by onsite general providers and referral to a psychiatrist for only the most complicated cases (45). Their on-site assessment rate improved from 10 to $84 \%$, and the number of mothers entering treatment improved from none to $90 \%$. They provide a practical training model for primary providers that could be adapted to a pediatric setting without need for additional mother-specific providers. Yawn et al's Translating Research into Practice for Postpartum Depression study was carried out in family medicine practices (50). Intervention sites received education and tools for postpartum depression screening and therapy initiation, including plans for follow-up visits, prescription support tools, and education about cognitive behavioral therapy and nursing follow-up calls to monitor medication initiation, adherence and side effects. Women in the intervention group were more likely to receive a diagnosis and therapy for postpartum depression, and tended to have lower depressive 


\section{Review | weiss-Laxer etal.}

symptom levels at 6 and 12 mo postpartum (50). Leung et al. (51) conducted a randomized controlled trial of structured screening using the Edinburgh Postnatal Depression Scale in Maternal and Child Health Centers in Hong Kong (which provide preventive care to mothers and children up to age 5 y), with control mothers receiving general clinical assessment (usual care). For this trial, clinic nurses were trained in "nondirective counseling" for maternal depression and received ongoing support from a community psychiatric team (to whom patients could also be referred). Both arms of the study had access to nondirective counseling or psychiatric team referral. Participants in the intervention arm were less likely to be above the study-established cutoff on Edinburgh Postnatal Depression Scale at 6 mo postpartum (51).

Finally, Kuosmanen et al. (41) described a different model in a clinic in Finland that provided primary care for both mothers and children. Although this model involved the expense of employing a new staff member-a mental health nurse-to conduct assessment and treatment, the primary care doctors also utilized a psychiatrist phone supervision service to manage more challenging cases, successfully incorporating most depression treatment within the mother and child clinic. This model highlights an advantage of delivering maternal and child health care in the same setting, as it eliminates one of the most fundamental barriers to maternal depression management in pediatrics.

The successful models in women's health care settings incorporate a comprehensive approach, with universal screening, substantial provider training (including in therapeutic strategies), mental health consultation and/or on-site mental health providers, and substantial support to ensure that referrals are completed (e.g. via nurse case managers). Many of the above interventions contain elements of collaborative care, a primary care-based model of mental health care with substantial empirical support for efficacy (52); that are expanding to include the obstetric setting (53). Core elements of collaborative care include: (i) joint definition of the problem (by the patient and care provider), (ii) joint development of a care plan, (iii) provision of support for effective self-management and training in affective, behavioral and cognitive change, and (iv) active, sustained follow up (53) and a caseload registry (54) which can help track patient progress and engagement. Factors found to be predictive of successful implementation of collaborative care programs or higher patient activation rates include strong leadership support, well-defined and implemented care manager roles, a primary care physician champion, an on-site and accessible care manager (54). Factors correlated with clinical outcomes (depression remission) included having an engaged psychiatrist, not seeing operating costs as a barrier to participation, and face-to-face communication (warm handoffs) between primary care practitioners and case managers (55). It is likely that many of these same ingredients are applicable to the management of maternal depression in pediatric settings.

Our search of the literature was systematic yet there are two limitations to this study. First, we searched English-language articles only and second, we did not use strict criteria to assess the methodological rigor of each study. Studies needed to report a systematic intervention and outcomes, but given the immature state of the science of managing maternal depression in pediatrics it was not possible to be more restrictive in the selection of studies.

\section{CONCLUSION: IMPROVING THE MANAGEMENT OF MATERNAL DEPRESSION IN PEDIATRICS}

Several issues merit consideration in creating an agenda for future development and research that can address the current shortcomings of pediatric interventions and incorporate lessons learned from other settings. We learned several lessons from the six pediatrics studies: First, pediatric provider training on maternal depression detection and management is necessary but insufficient. Second, supports are needed to prompt screening and track referrals, as several 'universal screening' programs encountered relatively low rates both of screening and of referrals of positive screens. There is potential for the electronic medical records to play a role in maximizing screening and referral rates. Third, on-site care coordinators dedicated to engaging mothers, interfacing with providers and assuring follow-up appears promising. From settings outside of pediatrics, we learned that elements of collaborative care (e.g., including active case management/care coordination, availability of brief on-site counseling and psychiatrist consultation), a well-studied model of integrated mental health care (defined by the Substance Abuse and Mental Health Services Administration as "the systematic coordination of general and behavioral healthcare"), have proven effective in obstetric and maternal health settings and should be considered for inclusion in pediatric-based models.

Specifically, practice recommendations include the necessity of training pediatric providers from a variety of allied health fields, such as medicine, nursing and social work, about maternal depression and related risk factors. In particular, the model of training residents and other health professionals during their clinical training offers the most potential for affecting long-term change in depression management. A potential approach to improving the management of mental health and other family risk factors in primary care pediatrics that shows promise but was not incorporated into any of the reviewed programs is that of training providers in a "common factors approach" (56). This involves ensuring providers have basic understanding of emotional problems and behavior change processes, can build positive expectancies, understand, and respond to the patient's and family's formulation of their distress, and can build therapeutic alliances-general skills that are common to successful management of a range of mental health problems. These skills can foster the ability of pediatric providers to manage not only the mental health concerns of their patients, but also those of their patients' parents.

In addition to the practice changes and issues highlighted throughout this paper, more rigorous study designs are essential to establish an evidence base for primary care pediatrics interventions that can effectively address maternal depression. A critical aspect for development and adoption of effective depression management in pediatrics is demonstrating the value of such programs to children's health, an aspect that is missing from 
all six models reviewed. Future studies would be well served to increase their follow-up time and to improve data collection measures in order to link maternal management programs with an evaluation of possible child health impacts (including outcomes such as developmental or behavioral problems, emergency room utilization, and timely receipt of well-child care, all of which have been found to be affected by maternal depression). In a 2012 review of postpartum depression management interventions in diverse settings, Yawn et al. (26) made a similar point, highlighting that current studies should be interpreted as preliminary and that longer follow-up studies are needed.

These models suggest the need to create better and more integrated mental health and primary care for all affected family members, at the levels of provider training and clinical practice. Ultimately, creating such systems would involve not just pediatric medical homes but family medical homes. Approaches of this type may be one of the most important steps toward addressing disparities in children's health.

\section{ACKNOWLEDGMENT}

The authors thank Lori Rosman, Public Health Informationist at the Welch Medical Library, for her assistance with the literature search.

\section{STATEMENT OF FINANCIAL SUPPORT}

N.S.W.-L. gratefully acknowledges support for her doctoral studies in public health from the C. Sylvia and Eddie C. Brown Community Health Scholarship Program.

Disclosures: We have no financial ties to products in the study or potential/ perceived conflicts of interest.

\section{REFERENCES}

1. Hay PJ, Ferrari AJ, Charlson FJ, et al. Burden of depressive disorders by country, sex, age, and year: findings from the Global Burden of Disease Study 2010. PLoS Med 2013;10:e1001547.

2. National Research Council and Institute of Medicine. Depression in Parents, Parenting, and Children: Opportunities to Improve Identification, Treatment, and Prevention. Committee on Depression, Parenting Practices, and the Healthy Development of Children. Board on Children, Youth, and Families. Division of Behavioral and Social Sciences and Education. Washington, DC: The National Academies Press, 2009.

3. Foster CE, Webster MC, Weissman MM, et al. Course and Severity of Maternal Depression: Associations with Family Functioning and Child Adjustment. J Youth Adolesc 2008;37:906-16.

4. Field T. Postpartum depression effects on early interactions, parenting, and safety practices: a review. Infant Behav Dev 2010;33:1-6.

5. Pilowsky DJ, Wickramaratne PJ, Rush AJ, et al. Children of currently depressed mothers: a $\mathrm{STAR}^{\star} \mathrm{D}$ ancillary study. J Clin Psychiatry 2006;67:126-36.

6. Murray L, Arteche A, Fearon P, Halligan S, Croudace T, Cooper P. The effects of maternal postnatal depression and child sex on academic performance at age 16 years: a developmental approach. J Child Psychol Psychiatry 2010;51:1150-9.

7. Shalowitz MU, Berry CA, Quinn KA, Wolf RL. The relationship of life stressors and maternal depression to pediatric asthma morbidity in a subspecialty practice. Ambul Pediatr 2001;1:185-93.

8. Center on the Developing Child at Harvard University. Maternal Depression Can Undermine the Development of Young Children: Working Paper No. 8, 2009. http://developingchild.harvard.edu/resources/reports_and_ working_papers/working_papers/wp8/.

9. Plant DT, Pariante CM, Sharp D, Pawlby S. Maternal depression during pregnancy and offspring depression in adulthood: role of child maltreatment. Br J Psychiatry 2015;207;213-20.

10. Wickramaratne P, Gameroff MJ, Pilowsky DJ, et al. Children of depressed mothers 1 year after remission of maternal depression: findings from the STAR*D-Child study. Am J Psychiatry 2011;168:593-602.
11. Hammen C. Depression Runs in Families: The Social Context of Risk and Resilience in Children of Depressed Mothers. New York: Springer-Verlag, 1991.

12. Silberg JL, Maes H, Eaves LJ. Genetic and environmental influences on the transmission of parental depression to children's depression and conduct disturbance: an extended Children of Twins study. J Child Psychol Psychiatry 2010;51:734-44.

13. Bronfenbrenner U, Morris PA. The bioecological model of human development. In: Damon W, Lerner R, eds. Handbook of Child Psychology. 6th edn. New York: Wiley and Sons, 2006.

14. Earls MF; Committee on Psychosocial Aspects of Child and Family Health American Academy of Pediatrics. Incorporating recognition and management of perinatal and postpartum depression into pediatric practice. Pediatrics 2010;126:1032-9.

15. Walker LO, Im EO, Tyler DO. Maternal health needs and interest in screening for depression and health behaviors during pediatric visits. J Pediatr Health Care 2013;27:267-77.

16. Olson AL, Kemper KJ, Kelleher KJ, Hammond CS, Zuckerman BS, Dietrich AJ. Primary care pediatricians' roles and perceived responsibilities in the identification and management of maternal depression. Pediatrics 2002;110:1169-76.

17. Olson AL, Dietrich AJ, Prazar G, et al. Two approaches to maternal depression screening during well child visits. J Dev Behav Pediatr 2005;26:169-76.

18. Chaudron LH, Kitzman HJ, Peifer KL, Morrow S, Perez LM, Newman MC. Self-recognition of and provider response to maternal depressive symptoms in low-income Hispanic women. J Womens Health (Larchmt) 2005; 14:331-8.

19. Liberto TL. Screening for depression and help-seeking in postpartum women during well-baby pediatric visits: an integrated review. J Pediatr Health Care 2012;26:109-17.

20. Silverstein M, Conroy K, Sandel M. Screening for social determinants of health in pediatric primary care. Pediatr Ann 2008;37:740-6.

21. Olson AL, Dietrich AJ, Prazar G, Hurley J. Brief maternal depression screening at well-child visits. Pediatrics 2006;118:207-16.

22. Gjerdingen DK, Yawn BP. Postpartum depression screening: importance, methods, barriers, and recommendations for practice. J Am Board Fam Med 2007;20:280-8.

23. Sockol LE, Epperson CN, Barber JP. A meta-analysis of treatments for perinatal depression. Clin Psychol Rev 2011;31:839-49.

24. Sockol LE. A systematic review of the efficacy of cognitive behavioral therapy for treating and preventing perinatal depression. J Affect Disord 2015;177:7-21.

25. Smith MV, Shao L, Howell H, Wang H, Poschman K, Yonkers KA. Success of mental health referral among pregnant and postpartum women with psychiatric distress. Gen Hosp Psychiatry 2009;31:155-62.

26. Yawn BP, Olson AL, Bertram S, Pace W, Wollan P, Dietrich AJ. Postpartum depression: Screening, diagnosis, and management programs 2000 through 2010. Depress Res Treat 2012;2012:1-9.

27. Bennett WL, Chang HY, Levine DM, et al. Utilization of primary and obstetric care after medically complicated pregnancies: an analysis of medical claims data. J Gen Intern Med 2014;29:636-45.

28. Chaudron LH, Szilagyi PG, Kitzman HJ, Wadkins HI, Conwell Y. Detection of postpartum depressive symptoms by screening at well-child visits. Pediatrics 2004;113(3 Pt 1):551-8.

29. Gjerdingen D, Katon W, Rich DE. Stepped care treatment of postpartum depression: a primary care-based management model. Womens Health Issues 2008;18:44-52.

30. Fernandez y Garcia EF, Joseph J, Wilson MD, et al. Pediatric-based intervention to motivate mothers to seek follow-up for depression screens: the motivating our mothers (MOM) trial. Academic Pediatr 2015;15:311-8.

31. Agapidaki E, Souliotis K, Christogiorgos S, et al. A theory-based educational intervention to pediatricians in order to improve identification and referral of maternal depression: a quasi-experimental study. Ann Gen Psychiatry 2013;12:37.

32. Wichman CL, Angstman KB, Lynch B, Whalen D, Jacobson N. Postpartum depression screening: initial implementation in a multispecialty practice with collaborative care managers. J Prim Care Community Health 2010;1:158-63. 
33. Katon WJ, Richardson L, Russo J, Lozano P, McCauley E. Quality of mental health care for youth with asthma and comorbid anxiety and depression. Med Care 2006;44:1064-72.

34. Feinberg E, Smith MV, Naik R. Ethnically diverse mothers' views on the acceptability of screening for maternal depressive symptoms during pediatric well-child visits. J Health Care Poor Underserved 2009;20:780-97.

35. Feinberg E, Smith MV, Morales MJ, Claussen AH, Smith DC, Perou R. Improving women's health during internatal periods: developing an evidenced-based approach to addressing maternal depression in pediatric settings. J Womens Health (Larchmt) 2006;15:692-703.

36. Dubowitz H, Lane WG, Semiatin JN, Magder LS, Venepally M, Jans M. The safe environment for every kid model: impact on pediatric primary care professionals. Pediatrics 2011;127:e962-70.

37. Feigelman S, Dubowitz H, Lane W, Grube L, Kim J. Training pediatric residents in a primary care clinic to help address psychosocial problems and prevent child maltreatment. Acad Pediatr 2011;11:474-80.

38. Dubowitz H, Kim J, Black MM, Weisbart C, Semiatin J, Magder LS. Identifying children at high risk for a child maltreatment report. Child Abuse Negl 2011;35:96-104.

39. Head JG, Storfer-Isser A, O'Connor KG, et al. Does education influence pediatricians' perceptions of physician-specific barriers for maternal depression? Clin Pediatr (Phila) 2008;47:670-8.

40. Carroll AE, Biondich P, Anand V, Dugan TM, Downs SM. A randomized controlled trial of screening for maternal depression with a clinical decision support system. J Am Med Inform Assoc 2013;20:311-6.

41. Kuosmanen L, Vuorilehto M, Kumpuniemi S, Melartin T. Post-natal depression screening and treatment in maternity and child health clinics. J Psychiatr Ment Health Nurs 2010;17:554-7.

42. Judd F, Stafford L, Gibson P, Ahrens J. The Early Motherhood Service: an acceptable and accessible perinatal mental health service. Australas Psychiatry 2011;19:240-6.

43. McDowell WK. Detecting women at risk for postpartum mood disorders. Nursing 2008;38:57-8.

44. Milgrom J, Holt CJ, Gemmill AW, et al. Treating postnatal depressive symptoms in primary care: a randomised controlled trial of GP management, with and without adjunctive counselling. BMC Psychiatry 2011;11:95.

45. Miller LJ, McGlynn A, Suberlak K, Rubin LH, Miller M, Pirec V. Now what? Effects of on-site assessment on treatment entry after perinatal depression screening. J Womens Health (Larchmt) 2012;21:1046-52.
46. Petrou S, Cooper P, Murray L, Davidson LL. Cost-effectiveness of a preventive counseling and support package for postnatal depression. Int J Technol Assess Health Care 2006;22:443-53.

47. O'Mahen H, Himle JA, Fedock G, Henshaw E, Flynn H. A pilot randomized controlled trial of cognitive behavioral therapy for perinatal depression adapted for women with low incomes. Depress Anxiety 2013;30:679-87.

48. Reay R, Matthey S, Ellwood D, Scott M. Long-term outcomes of participants in a perinatal depression early detection program. J Affect Disord 2011;129:94-103.

49. Kingston D, Austin MP, Hegadoren K, et al. Study protocol for a randomized, controlled, superiority trial comparing the clinical and cost- effectiveness of integrated online mental health assessment-referral-care in pregnancy to usual prenatal care on prenatal and postnatal mental health and infant health and development: the Integrated Maternal Psychosocial Assessment to Care Trial (IMPACT). Trials 2014;15:72.

50. Yawn BP, Dietrich AJ, Wollan P, et al.; TRIPPD practices. TRIPPD: a practice-based network effectiveness study of postpartum depression screening and management. Ann Fam Med 2012;10:320-9.

51. Leung SS, Leung C, Lam TH, et al. Outcome of a postnatal depression screening programme using the Edinburgh Postnatal Depression Scale: a randomized controlled trial. J Public Health (Oxf) 2011;33:292-301.

52. Katon W, Unützer J, Wells K, Jones L. Collaborative depression care: history, evolution and ways to enhance dissemination and sustainability. Gen Hosp Psychiatry 2010;32:456-64.

53. Grote NK, Katon WJ, Lohr MJ, et al. Culturally relevant treatment services for perinatal depression in socio-economically disadvantaged women: the design of the MOMCare study. Contemp Clin Trials 2014;39:34-49.

54. Whitebird RR, Solberg LI, Jaeckels NA, et al. Effective Implementation of collaborative care for depression: what is needed? Am J Manag Care 2014;20:699-707.

55. Eckstrom J, Williams D, Avery M, Unützer J. The utility of a caseload registry: perceptions of behavioral health clinicians working in an integrated primary care and mental health program. Gen Hosp Psychiatry 2015;37:329-34.

56. Brown JD, Wissow LS. Rethinking the mental health treatment skills of primary care staff: a framework for training and research. Adm Policy Ment Health 2012;39:489-502. 